\title{
Designing for Exploratory Play with a Hackable Digital Musical Instrument
}

\author{
Andrew P. McPherson \\ Centre for Digital Music \\ Queen Mary University of \\ London \\ London, UK \\ a.mcpherson@qmul.ac.uk
}

\author{
Alan Chamberlain \\ Mixed Reality Laboratory \\ The University of Nottingham \\ Nottingham, UK \\ alan.chamberlain@ \\ nottingham.ac.uk
}

\author{
Adrian Hazzard \\ Mixed Reality Laboratory \\ The University of Nottingham \\ Nottingham, UK \\ adrian.hazzard@nottingham.ac.uk
}

\author{
Sean McGrath \\ Mixed Reality Laboratory \\ The University of Nottingham \\ Nottingham, UK \\ sean.mcgrath@nottingham.ac.uk
}

\author{
Steve Benford \\ Mixed Reality Laboratory \\ The University of Nottingham \\ Nottingham, UK \\ steve.benford@nottingham.ac.uk
}

\begin{abstract}
This paper explores the design of digital musical instruments (DMIs) for exploratory play. Based on Gaver's principles of ludic design, we examine the ways in which people come to terms with an unfamiliar musical interface. We describe two workshops with the D-Box, a DMI designed to be modified and hacked by the user. The operation of the D-Box is deliberately left ambiguous to encourage users to develop their own meanings and interaction techniques. During the workshops we observed emergent patterns of exploration which revealed a rich process of exploratory play. We discuss our observations in relation to previous literature on appropriation, ambiguity and ludic engagement, and we provide recommendations for the design of playful and exploratory interfaces.
\end{abstract}

\section{Author Keywords}

Ludic design; ambiguity; musical instruments; exploration; play; hacking.

\section{ACM Classification Keywords}

H.5.2 User Interfaces: Evaluation/methodology; H.5.5 Sound and Music Computing: Methodologies and techniques, Systems

\section{INTRODUCTION}

Traditional HCI principles developed for workplace tools, including metrics of accuracy, efficiency and clarity, do not capture the whole spectrum of human interaction with technology. Domains such as the home $[11,23,30]$ and interactive

Paste the appropriate copyright statement here. ACM now supports three different copyright statements:

- ACM copyright: ACM holds the copyright on the work. This is the historical approach.

- License: The author(s) retain copyright, but ACM receives an exclusive publication license.

- Open Access: The author(s) wish to pay for the work to be open access. The additional fee must be paid to ACM.

This text field is large enough to hold the appropriate release statement assuming it is single spaced.

Every submission will be assigned their own unique DOI string to be included here. digital art [29, 43, 42, 41] present different opportunities and challenges, particularly as technology use in these domains is often less goal-directed, more open-ended and more personal.

Ludic design is a term coined by Gaver [21] to describe playful interactions with technology where the intrinsic experience of using a system is privileged over traditional metrics of utility. Ludic design seeks to be "richly suggestive ... without implying preferred interpretations". It seeks to promote curiosity and exploration [24], values ambiguity and openness to interpretation [23], and provides the capacity for appropriation by the user $[28,13]$.

This paper explores ludic design and evaluation principles in the context of digital musical instruments (DMIs). It presents two workshops using the D-Box [63], a new DMI which is designed to be appropriated, modified and hacked by the performer. The function and meaning of the D-Box is deliberately left open to interpretation to encourage the user to develop personal uses for the instrument that the designer may not have anticipated.

After a survey of the background and context, we introduce the design principles of the D-Box in relation to appropriation and playful interaction. We then describe the format of the workshops, which examined how users come to terms with an unfamiliar instrument through exploratory play. Workshop outcomes included: a distinctive pattern of exploration which differed from what previous literature has observed in software tools and interactive art; the presence of creative tension around whether participants felt in control of the hacking process; and the emergence in several participants of a sense of personal attachment to their hacks. We discuss the implications of these findings for playful and exploratory design. 


\section{BACKGROUND}

\section{Work and Play in Musical Instrument Use}

Digital musical instruments would seem to be a natural vehicle for studying playful interaction within HCI. Most musicians do not aspire to become professionals, and many do not perform in public at all. Rather, the experience of playing an instrument can be its own reward. Jordà [31] highlights the evolving nature of the performer-instrument relationship and the central importance reaching of the "rewarding point" in the learning process. DMI design for non-specialist users is a significant research area, especially in collaborative or public settings, e.g. [5, 55, 4].

Nonetheless, perhaps surprisingly, the exchange of ideas between DMI design and HCI has more often followed a different path, applying task-based HCI metrics to DMI evaluation $[59,33]$ or evaluating aspects of control and learning curve [31]. It is widely accepted that control accuracy alone is not a sufficient metric, leading to investigations of DMI "expressiveness" $[49,14,32]$.

Implicit in these evaluations is the notion that the DMI is, if not a strictly professional tool, then at least a goal-oriented one, whose goal is performance (whether public or private). The degree to which the musician can successfully perform (or express themselves) becomes the metric of an instrument's success. This is undeniably important, especially in connecting to existing expert communities [38], but it is only part of the picture. It is also not to say that DMIs have only one interpretation. There is recognition of competing stakeholders in an artistic situation [46], and the way meaning and use shift over time has been explored in longitudinal studies [37, 25, 38].

Still, there remains an opportunity to specifically examine the early stages of encountering an unfamiliar DMI: playing in the ludic rather than musical sense. How people come to understand an instrument's meaning has implications not only for possible later performance, but for a broad range of HCI domains.

\section{Appropriation, Hacking and Making}

The history of musical instrument use is closely bound up with the phenomenon of appropriation [44, 16], where the user develops a personal working relationship with the tool that may differ from the designer's intent. Transformative musical techniques such as electric guitar distortion and DJ turntable scratching emerged from musicians exploiting engineering limitations in unexpected ways. Capacity for appropriation has been suggested as an HCI design goal [30, $28,13]$ which is closely bound up with encouraging ambiguity [23], openness to interpretation [52], and even unfinished designs [53].

On the other hand, it is inherent in the nature of appropriation that unexpected uses may emerge whether or not the designer seeks them out. Openness also does not imply that tools should be more complex. Though in interactive art, Morrison [43] finds that simple behaviours lead to boredom amongst experts, within music the creative benefit of constraints is well-established [27, 36], and may in fact encourage unusual appropriations of music technology [64]. Dahlstedt et al. [12] suggest the value of simple tools with open-ended musical interpretations in group improvisation.

Roedl et al. [50] examine the recent shift in HCI discourse from "user" to "maker", arguing that a certain resourcefulness characterises the maker identity: "makers are not satisfied using products exactly as they are designed. Rather, makers are motivated by a desire to adapt, customize, and improve on technology in order to better suit their particular goals and tastes." Tanenbaum et al. identify playfulness as a characteristic of maker culture, while still being aligned with utility [56]. Hackspaces and similar community hubs have been an important source of innovative new hardware products [35]. These trends collectively suggest that appropriation, modification and completion of ambiguous or unfinished designs, and design of new systems may represent points along the same spectrum of creative activity.

\section{Circuit Bending}

Circuit bending $[26,9,10]$ is both the direct inspiration for the D-Box and an interesting example of the space between appropriation and design. Through exploratory rewiring, circuit benders transform toys, mass-market music equipment and other consumer electronics into new audio or visual media pieces. The intended outcome may not be known in advance, and both systematic and playful experiments may contribute to the result. Similar processes can be applied to physical objects as exemplified by Bowers' infra-instruments [7].

\section{Learning and Playing}

The role of learning in the context of technology has been well documented. Learning is formative [34]. There are many techniques that individuals rely on to assimilate knowledge and engage with new experiences $[54,51,2,20]$.

Our D-Box workshops, whose format is described in the next section, represent a hybrid approach utilising guided [60], semi-guided [47] and at times unguided learning [1] to provide an initial entry point of access without relying on goaloriented tuition. The workshops support a process of expansive learning by offering one-to-one support in helping learners to achieve their goals [18]. This way, learners can define their own agenda and progress towards it, without first being told how they should interact with the devices. For familiar instruments, famous performers, tutorial materials and personal experience may mean that the first hands-on encounter is already goal-directed; the status of the D-Box as a novel instrument reduces these biases.

Bloom offers the view that systematic learning can lead to mastery through a series of increasingly challenging interactions [6], but limited time and scope means that these workshops are not designed to be iterative learning assignments to develop conceptual understanding, but rather experiential engagements where people learn and develop initial skills.

\section{THE D-BOX}

The D-Box (Figure 1 top) is a self-contained digital musical instrument expressly designed to be modified and hacked by the user [63]. 


\section{Form and Appearance}

The instrument consists of a $15 \mathrm{~cm}$ wooden cube with two $2 \times 10 \mathrm{~cm}$ capacitive touch sensors on the top surface, each of which reads the location and contact area of up to 5 fingers. Inside the cube is a $10 \mathrm{~cm}$ speaker at the front, a rechargeable battery and a BeagleBone Black single-board computer which handles audio and sensor data processing [39].

We seek to leave the interpretation of the D-Box open to the user, without pushing them strongly toward any existing instrumental paradigm. The openness allows observation of how people come to terms with unfamiliar music technology. To this end we did not engage with user-centred or participatory design approaches, which seek to align the designers' and users' interpretations from the initial point of creation, and the D-Box design deliberately avoids obvious metaphors with other acoustic or digital instruments. The $15 \mathrm{~cm}$ size is just small enough to fit in one hand, but large enough to be more comfortable in two. The cube shape allows for a wide range of orientations, playing techniques and postures, which tend to vary between users [64]. The touch sensors on the surface are an original design whose appearance and function will not be familiar to most users.

Previous work has shown that constrained musical instruments often encourage users to develop creative playing techniques $[36,27,64]$. For this reason, the action-sound mapping of the D-Box is deliberately simple and limited at first: one touch sensor controls the pitch of the sound, while the other controls the centre frequency of a bandpass filter effect. In our workshops, it is initially left to each user to discover which actions have a meaningful effect on the output sound.

\section{Hacking and Exploration}

Removing the side panel of the box reveals a solderless breadboard (Figure 1 bottom) known as the matrix, containing a collection of simple analog circuits. In total, 8 analog inputs and 8 analog outputs connect from a custom expansion board on the BeagleBone to the matrix. The breadboard is prepopulated with resistors and capacitors which form a collection of idiosyncratic feedback loops [40] which collectively govern the behaviour of the instrument.

All circuits on the matrix can be reconfigured by the user, and there are no restrictions on what connections can be made nor what components can be used. 220 -ohm protection resistors on the board prevent damage from shorting outputs or power connections together [63].

Once the performer encounters the matrix, the initially simple and constrained mapping gives way to a much broader space of possibilities obtainable by changing the wiring (referred to in this paper as "hacking"; see [63] for a discussion of this term). However, as compared to standard modular synth approaches using control voltages (CVs), changing the feedback circuits can produce unusual, even chaotic results, though the results are generally deterministic and repeatable [40]. Avoiding familiar CV approaches was a deliberate design decision aimed at encouraging the performer to discover unique personal configurations that may diverge significantly from the designer's original intent.

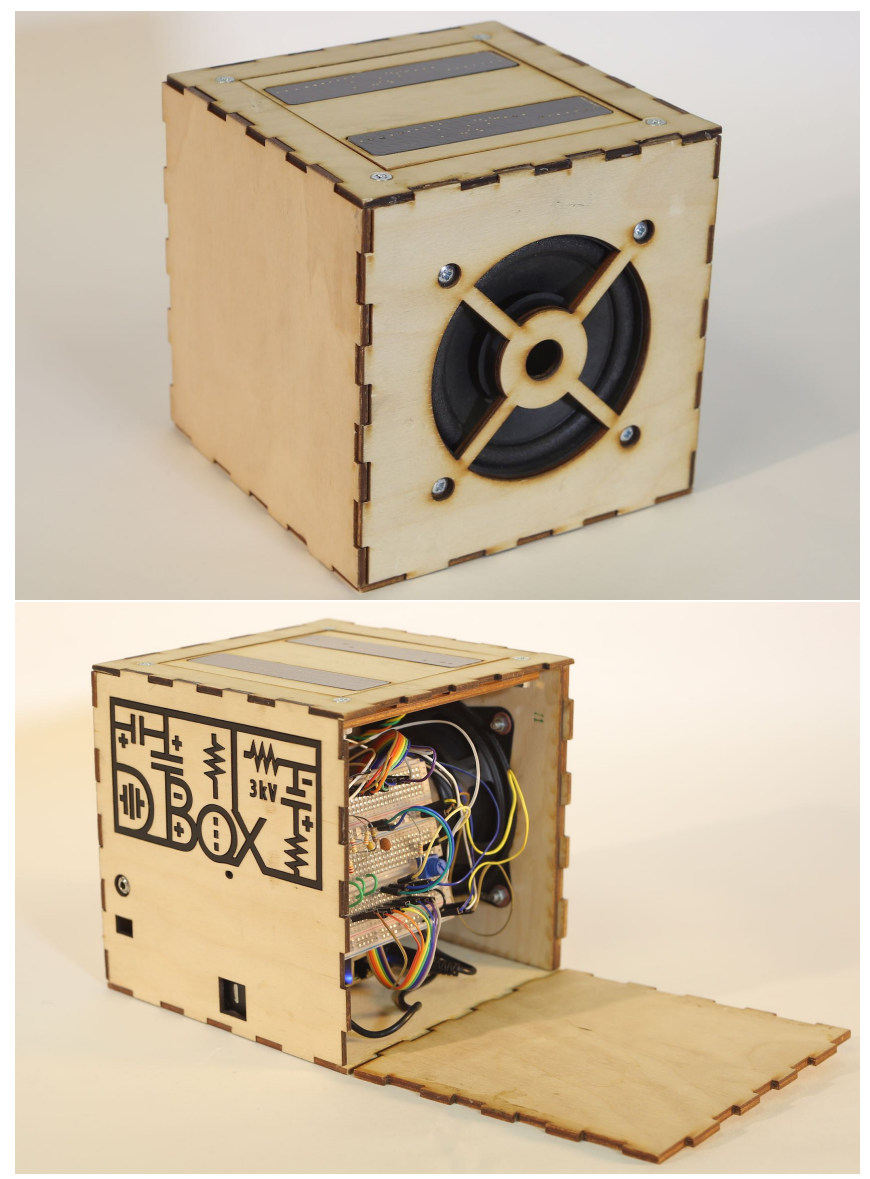

Figure 1. The D-Box. Top: exterior view, showing touch sensors (top surface) and speaker. Bottom: interior view, showing the default state of the breadboard (matrix) for hacking the instrument.

An important byproduct of the D-Box design is that without either significant prior experience of working with the D-Box or a detailed user manual and significant time to read it, goaldirected exploration of the matrix is difficult or impossible. As in circuit bending [26], it will not be immediately obvious which circuit controls which feature, nor what the effect of a given change might be. Thus the D-Box deliberately seeks ambiguity [23] and encourages a divergence of interpretation. We seek to design for appropriation $[28,13]$ where we expect the user to adopt the tool in an idiosyncratic fashion, without predicting or deliberately biasing what that interpretation may be. It may be fair to describe the designer-user relationship as adversarial: the space of possibilities is deliberately nonlinear and opaque.

\section{Previous Use}

In previous work, the D-Box was used in a performance study with 10 musicians, each of whom worked with a D-Box over several weeks to produce two performances. We observed a wide variety of usage patterns and hacking techniques which are explored in $[63,40]$. Amongst the observations were a diverse variety of physical modifications to the box and the circuits within, and contrasting approaches between hacking- 
as-preparation (designing an instrument to be used in performance) and hacking-as-performance (where the instrument is modified live on stage). Several study participants have continued to use the D-Box in their personal performances.

In this study, instead of focusing on the results of long-term engagement, we examine the process by which lay users come to terms with the affordances and constraints of the DBox over a period of several hours. The status of the D-Box as an unfamiliar technological object without clear correct and incorrect uses offers a window into exploratory play. Comparison between exploratory play and long-term performance studies will be further addressed in the Discussion section.

\section{D-BOX HACKING WORKSHOPS}

\section{Format}

We staged two hacking workshops, each a half day long. Both workshops were free, open to the public, and targeted to adults with or without musical experience. Workshop 1 was held at an established art technology venue in Liverpool, UK. 6 participants ( 3 female) took part, with all participants sitting around one large circular table. Workshop 2 was held at a community centre in London. 11 participants (6 female) were split across three small rectangular tables (4, 4 and 3 people) in the same room.

For both workshops, no technical background was required, though a few participants had previous music technology experience (see Table 1). No participants had previously worked with the D-Box, though some had seen a YouTube video containing several short performance excerpts which was used in email advertising for the event.

The workshops were led by one of the authors, who explained each step and assisted participants with their D-Boxes when needed. The workshops thus mixed guided learning (tutorials from leader), semi-guided learning (when participants actively sought help) and unguided learning (which compromised the bulk of the hacking process).

\section{Stages}

Both workshops followed a similar format. At the beginning, each participant received their own D-Box in its default configuration, with the wooden panels closed, obscuring the breadboard. Participants were asked to learn to play it, having been given no information beyond how to turn it on. The purpose of this stage was to capture the ways in which participant made meaning from an unfamiliar tool.

After a 10-15 minute period of initial exploration, participants then were instructed to open the box and examine the breadboard inside. The bulk of the workshop from this point forward (2-3 hours) focused on hacking inside the box, where modifying the breadboard circuits altered the behaviour of the instrument. At this point, jumper wires and small electronic components were provided at each table for participants to use in their hacks. In Workshop 1, an arbitrary selection of components, mainly consisting of resistors and capacitors, was placed near each group of participants; in Workshop 2,

\begin{tabular}{|l|l|l|}
\hline \multicolumn{3}{|c|}{ Workshop 1 } \\
\hline P1 & Male & Student of Music Tech \\
\hline P2 & Male & $\begin{array}{l}\text { Film maker, non-musician, experienced 'maker' of } \\
\text { mechanical objects }\end{array}$ \\
\hline P3 & Male & $\begin{array}{l}\text { Student, non-musician, limited experience of digital } \\
\text { technology }\end{array}$ \\
\hline P4 & Female & $\begin{array}{l}\text { Artist and composer working with traditional } \\
\text { instruments. Limited experience of digital music } \\
\text { making }\end{array}$ \\
\hline P5 & Female & $\begin{array}{l}\text { Artist and DJ, experience with live performance and } \\
\text { playing percussion. }\end{array}$ \\
\hline P6 & Female & $\begin{array}{l}\text { Student, non-musician, limited experience of digital } \\
\text { technology }\end{array}$ \\
\hline P7 & Female & $\begin{array}{l}\text { Classical music student, emergent understanding of } \\
\text { music technology }\end{array}$ \\
\hline P8 & Female & Academic, some music technology experience \\
\hline P9 & Male & Student, plays drums, no music technology experience \\
\hline P10 & Male & $\begin{array}{l}\text { PhD Student, experience in acoustic instruments and } \\
\text { music technology }\end{array}$ \\
\hline P11 & Male & Student, non-musician, some technical background \\
\hline P12 & Male & $\begin{array}{l}\text { Student, some musical experience, no reported } \\
\text { technical experience }\end{array}$ \\
\hline P13 & Female & $\begin{array}{l}\text { Some technical exposure (signal processing and } \\
\text { electronics), plays a musical instrument. }\end{array}$ \\
\hline P14 & Female & $\begin{array}{l}\text { Therapist with a focus on brain injuries and learning } \\
\text { difficulties. Self-reported as "not very technical." }\end{array}$ \\
\hline P15 & Male & $\begin{array}{l}\text { Sound engineer, some experience with Arduino, } \\
\text { analogue synths }\end{array}$ \\
\hline P16 & Female & $\begin{array}{l}\text { Some experience with electronics e.g. repairing } \\
\text { laptop, learning piano }\end{array}$ \\
\hline P17 & Female & $\begin{array}{l}\text { Intern, did electronics degree (radio and sensors), } \\
\text { experience in software-based music, plays several } \\
\text { instruments. }\end{array}$ \\
\hline
\end{tabular}

Table 1. Participants in the D-Box workshops.

components were located in the centre of each table. In practice we observed that wires were used most commonly, followed by capacitors and then resistors.

For most of the hacking time, participants were free to explore as they saw fit. The period was punctuated by interventions from the workshop leader to maintain interest and direction: first, participants were pointed to online documentation on the D-Box along with a video of performances, both accessible by mobile phone. Subsequently, the workshop leader described a series of example hacks and gradually explained the function of a few of the breadboard circuits (less than 50\% in total). These examples were intended to provide inspiration for the participants in their own hacks, not to provide a systematic tutorial of the D-Box nor to define certain hacking processes as right or wrong. Similarly, when answering questions, the workshop leader attempted to provide focused guidance without suggesting that certain hacks or processes were better than others.

In the last hour of the workshop, participants were directed to exchange D-Boxes. This meant that each participant received a D-Box hacked according to a process they had not witnessed. In Workshop 1, participants knew the identity of the person who they had received the D-Box from; in Workshop 2, boxes were rotated between tables so the participants did not know who it came from. Although participants could have used the same D-Box for the entire workshop, the ex- 


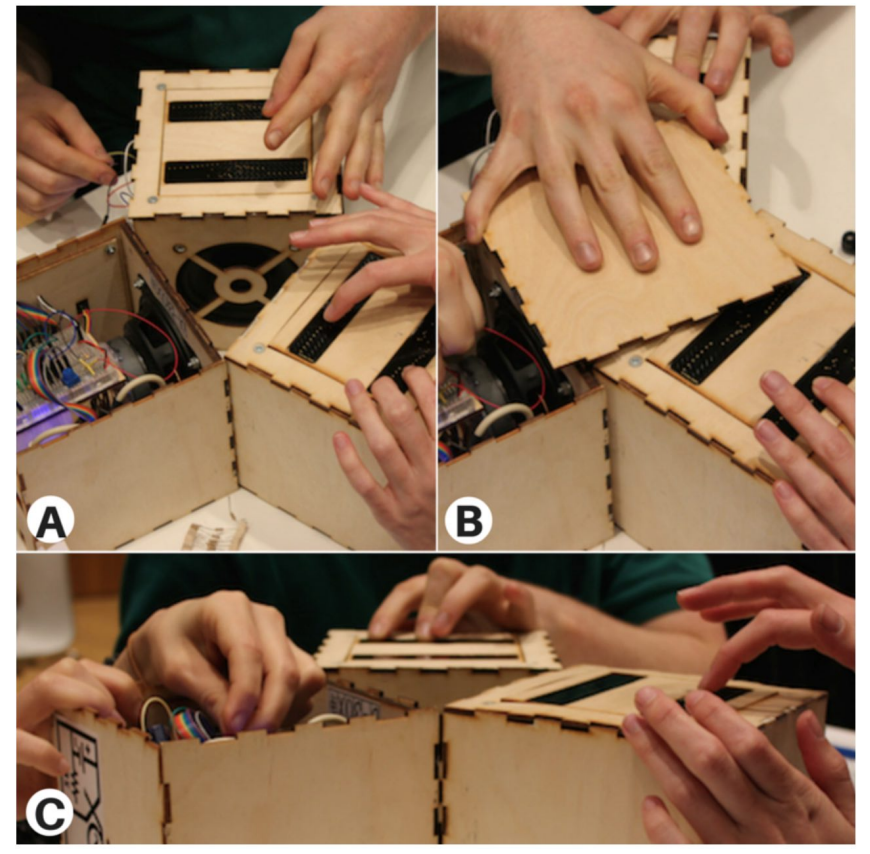

Figure 2. P15, P16 and P17 give a group D-Box performance, with the wooden side plate reused to mute the speakers in the centre of the triangle.

change was a deliberate attempt to perturb the hacking process and observe how the participants reacted.

After a short period exploring and playing the new D-Box, participants were given the opportunity to develop a further, final hack that they were then invited to perform to the group at the end of the workshop. In Workshop 1, participants showed their final hacks but none gave a prepared performance. In Workshop 2, each table chose to perform together (see Figure 2 for one example).

\section{Data Collection}

Aside from the workshop leader, a team of researchers (4 for $\mathrm{W} 1 ; 3$ for W2) captured video documentation of the workshop including observations of the participants' hacking interactions and periodic interviews at the culmination of the stages of the workshop, inviting the participants to explain their hacks and their processes as they went along. Each participant was also interviewed following the end of the workshop. Interviews served to clarify observations during the workshop, establish the background of the participant, and gather their final thoughts on the D-Box.

The workshop format was intended to capture a detailed qualitative picture of each participant's actions, reactions and remarks. It was expected that each participant might bring their own idiosyncratic approach to hacking and exploration, and our documentation prioritised capturing individual actions and thought processes in detail rather than attempting comparisons on any numerical metric. Following the workshop, interviews and comments were transcribed and grouped into themes.

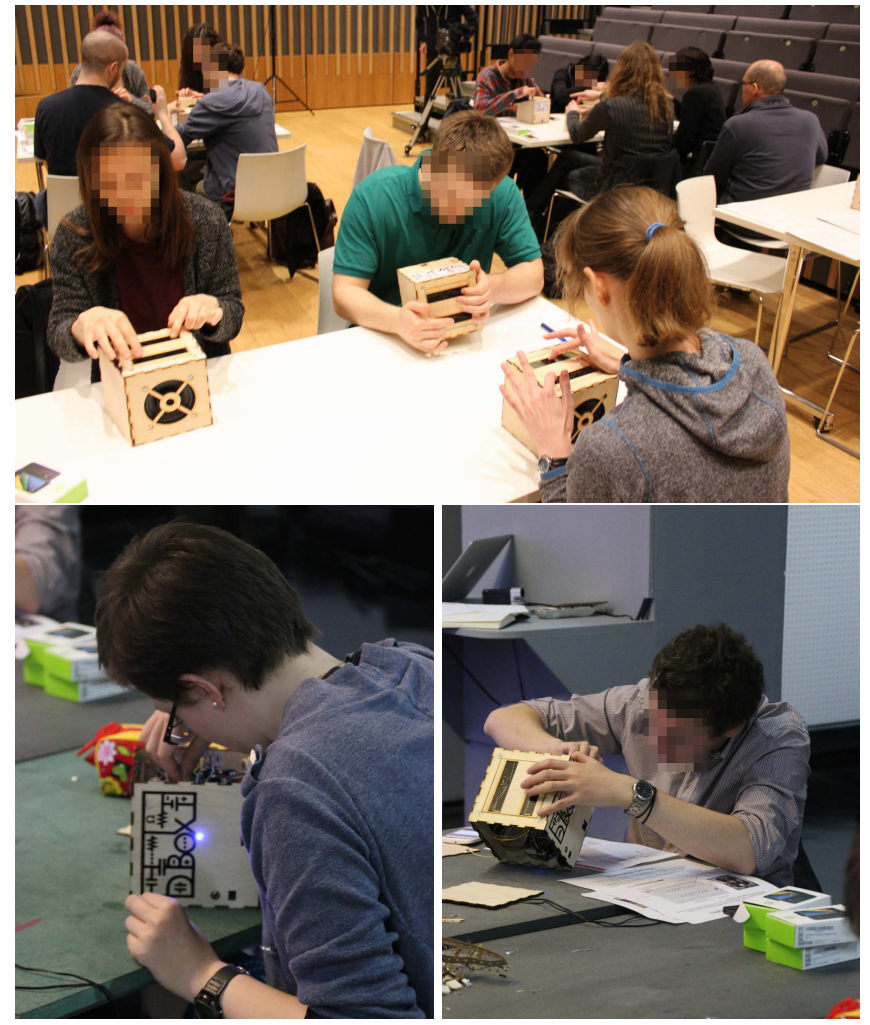

Figure 3. Top: Workshop 2 during the initial encounter with the D-Box. Bottom: two participants from Workshop 1 hacking the D-Box.

\section{WORKSHOP OBSERVATIONS}

This section presents the most salient observations from the two workshops, focusing particularly on three periods of activity: the participants' first encounter with the D-Box (Figure 3 top); their process of making hacks (Figure 3 bottom); and the reactions to participants exchanging D-Boxes with one another. Participants are identified by number (P1-P17: P1-P6 represent workshop 1, P7-P17 workshop 2) in the following text; refer to Table 1 for details. The design implications of these observations are then addressed in the Discussion section.

\section{First Contact with the D-Box}

In this phase of the workshop, we sought to understand how participants discovered the affordances of the D-Box having been given no instructions. Each participant typically began by inspecting the six faces of the cube, after which attention focused on the two black touch sensor strips on the top of the box. Each person quickly worked out that one sensor controls the pitch of the sound and proceeded to explore the details of its operation. Several playing techniques were observed, including tapping, holding and stroking along the sensor surface with the fingers and touching keys and mobile phones to the sensor.

The physical design of the sensor guided participants' expectations about its operation, and these expectations were not always fulfilled. P6 noticed the exposed vias on the sensor circuit board (Figure 1 top) and surmised that they held spe- 
cial significance: "I came up with hypotheses: do the sounds change when you are different distances from the knobs... experimented a few times. Nothing changed, hypothesis rejected."

Participants also developed playing techniques by observing their neighbours. P6 commented: "I saw someone else start stroking [the sensors] and I thought, I'll do that as well." P6 discovered the pressure capability and demonstrated it to P4 and P5: "if you press harder it gets louder." P5 discovered that hovering a finger just about the sensor would activate it, and demonstrated the technique to P4 and P6: "you don't need to touch it for it to work."

While all participants understood the pitch sensor, the second sensor (controlling a bandpass filter on the sound) was not well understood. P1, a music technology student, worked it out: "one's a pitch and one's a filter." But P2's reaction was typical of several participants: "I found that one just sort of cut the other one out in some ways." P7 and P8 believed that this sensor strip was nonfunctional.

P6 discovered that touching the speaker cone created a damping effect on the sound. Otherwise, very few playing techniques emerged using anything other than the two sensors.

\section{Producing and Exploring Hacks}

Once initial exploration finished, participants were invited to open the D-Box and inspect the breadboard within. Perhaps reflecting a general reaction to electronic circuits, at least two participants asked whether it was safe or acceptable to touch inside the box, e.g. P3: "if you touch these [wires] together is that going to blow up?" Participants were assured that there was no safety hazard nor was there any way to damage the box by rewiring it. Other participants attempted to use visual cues on the breadboard to guess at its function. P5: "I'm very interested in how sound and colour correlate, and I'm happy to see that the colours inside are rainbow colours. So each sound has a colour, is that right?"

\section{Discovering New Hacks}

The process of finding new hacks typically began by following the instructions of the workshop leader (P9 explains one of his hacks as "I'm doing what he asked us to do") or bringing in assumptions from prior knowledge (P5's focus on colours; P10's experience with the software tool Max/MSP).

Some participants initially attempted to develop a systematic understanding of the breadboard function. However, the design of the D-Box, while deterministic in behaviour, is also deliberately resistant to easy analysis. This led participants to shift their approach. P1 commented: "I was trying to be really methodical with it and it didn't work very well for me. It lends itself well to being a lot more experimental." Indeed, 11 of the 17 participants specifically commented on taking a trial-and-error approach to developing hacks, and no participant mentioned retaining a systematic approach. P6 explained: "I was experimenting in a systematic way and then I was like 'nah!' I decided to move things around in a bit of a random way and see what happens. I just went a bit mental with it and I'm here now." P10: "I don't really get what I'm doing precisely, so I'm just experimenting basically." P17:
"I've abandoned any sort of method and I'm just doing random." P9 explained that he understood what he was doing " 60 , 50 percent."

Despite the experimental approach, several participants remained confident that a systematic understanding was possible. P2: "It creates sounds and seems to me to be slightly out of control ... but I don't think it is." At least four participants commented that with more time or a different situation, they would take a systematic approach to hacking the D-Box. P16 commented: "I think if I was by myself I'd work at it in a systematic manner, it's different with lots of people around, so I'm just messing around."

Caution was a characteristic common to many participants' approaches (and commented on by P6, P8, P9, P12). P9 explained that he took a maximum of two steps forward before evaluating his hack. P6 explained the process as: "move one wire ... move it back." The tension between discovering a new hack and saving or restoring previously favoured hacks is further addressed in the next subsection and in Discussion.

In general, self-described reactions to the hacking process included curiosity (P3, P10), playfulness (P17), excitement (P12, P14, P17) and sometimes frustration (P11, P13). Frustration emerged particularly from lack of sonic variety, and was typically a transient state associated with particular breadboard configurations. P13 commented: "I can't make it do very many things. I guess I'm kind of frustrated that I can only make it play one type of sound at the same time." Notably, none of the 17 participants disengaged from the activities or left the workshop early (including during the lunch break which would have been a natural breakpoint).

Most of the favourite hacks identified by participants resulted from exploratory or arbitrary actions. P13 demonstrated a sound: "I put the impact sensor from the purple to the blue. But the blue is in the yellow row. I don't know what that means, but it does this!" Later, P13 commented: "The ones I enjoyed sonically was the one that was just me not quite plugging things in."

The D-Box is designed to stop making sound within a few seconds of removing the fingers from the touch sensors. If necessary, the instrument could be forcibly silenced by powering it off. No participant was observed doing this during the workshop. In one case, P14 produced a hack where sound continued after removing the fingers. She reacted with enthusiasm: "I made it do something without me doing anything. That was fun. It's so cool."

Technological failure happened infrequently. When it occurred, it was a source of temporary frustration or embarrassment, but taken in stride (sometimes with assistance from the workshop leader). In one exchange, P12 and P14 were laughing at their sounds. P14's D-Box then stopped working. Asked what happened, P14 replied: "I have absolutely no idea. I fiddled and it went kaboom!"

\section{Saving or Reverting Hacks}

Perhaps the most interesting part of the hacking process was the point when a participant converged on a sound they liked. 
After often arbitrary exploration, participants would become increasingly cautious and systematic as they approached a desired result. P6 explained: "I just randomly tended to re-plug things in ... but once it got to a stage where I quite like the sound, it was no longer random, so I wanted to control the sound and manipulated it in a way I wanted to achieve a certain outcome."

As a hardware system, the D-Box breadboard has no builtin undo feature aside from manually reverting to a previous wiring configuration. There is no way to save global presets, though P7 and P9 requested this capability. This led to tension between maintaining a favoured hack and continuing exploration, as participants weighed the costs and benefits of losing the state they had gotten to. P8: "There's a moment where you want to go, 'oh, don't go too far' because you want to get back to that nice sound I had."

Participants relied on memory to backtrack if a modification didn't produce a desired result (P1, P12; P6: "if it made the wrong sound, I'd make it go back."; P8: "just try and remember the steps I've taken"). P9 explained that if he did not like a result, "I turn back, I go to the original state and change something else." Being unable to recreate a previously desired hack was a common experience (P10, P13, P14, P16; P4: "I found some other favourites earlier but I can't find them again."). Participants cited this experience as a reason for taking a cautious approach. Surprisingly, no participants made written notes through several mentioned they could (P4, P16, P17). When asked why, most participants mentioned the short duration of the workshop. P15 suggested the use of a mobile phone to take a picture of desired breadboard states.

As in the initial encounter with the D-Box, social factors emerged in backtracking from an undesirable hack. In one exchange, after P13 makes an undesired change, P14 asks: "You took the resistor out. Where did you take it out from?" P13: "I don't know." P14: "Was it here?" P13 pauses, then: "Yeah." P14 reconnects the resistor in P13's box. A similar exchange was observed between $\mathrm{P} 7$ and $\mathrm{P} 9$ over where a lost part should go. (Both P7-P9 and P13-P14 knew one another before the workshop and arrived together.) Generating new hacks tended to be an individual rather than group process.

\section{Exchanging Hacks}

In the latter part of the workshop, in a deliberate attempt to perturb the hacking process, the participants exchanged hacked D-Boxes. A surprising result was the strong sense of disappointment expressed by many participants at their new instrument (P3, P11, P12, P13, P16). P11 commented of the new D-Box: "It doesn't make much sound. This is sad.... This box has been mistreated." P16 likewise described the new D-Box as "a bit sad". P13 compared her new instrument unfavourably to nu metal music. P12 commented: "I don't like it. It doesn't do anything. It needs changing."

In a similar vein, several participants (P3, P12, P14) commented that they missed their old D-Box. P14: "I miss mine [the old box]. I knew exactly what I'd done." P12: "I liked my box. It's the configuration isn't it? Having done your own hack, it does feel very personal." Some participants further identified a sense of personal invasion associated with working with someone else's D-Box. (P2, P12; P11 commented: "It feels wrong to touch someone else's box," to which P14 agreed: "Yeah, it feels weird.")

P12 cast judgment on the previous user of the D-Box: "I'm not sure they knew what they were doing. I mean, neither did I, but at least I produced some sounds!" Collectively, these reactions suggest the emergence of a sense of personal connection or ownership, despite the self-acknowledged undirected hacking process. This phenomenon will be further explored in Discussion below.

Not all participants disliked the new D-Boxes. P4, P5 and P17 liked the new ones (P4: "Oh, it's well better to be honest! I got a little bit lost with mine"), as did P9 whose old D-Box had gone silent. P6 and P15 had more neutral reactions to the swap.

After the swap, many participants expressed that they felt they'd lost control and understanding of the hacking process (P2, P5, P7, P8, P9, P10, P14). P9 explained: "I guess it's like, you've made the changes yourself $[\mathrm{P} 7$ nods in agreement] and you've made a conscious decision when you made the change 'I like this sound', so that's why I feel more in control. For this one, you're just given a random thing and you don't know what's going on." Perhaps as a result, a qualitative change appeared in the hacking process with many participants taking a more aggressive, less cautious approach. P12: "I'm being braver on what I'm trying out on the breadboard because it's already been destroyed!"

$\mathrm{P} 2$ created one of the most aggressive hacks in the new DBox, pulling out all the wires and letting them dangle free within the box. He then added a random collection of electronic components and an electric motor from a dismantled toy he had brought with him (Figure 4). When the motor spun, it caused transient connections within the pile of components, creating a chaotic noise. P2 explained: "I can't logically play this thing, so I literally took it apart and tried to make it play itself. I didn't feel very in control, so I decided to give that control to something else."

\section{Reflections on the D-Box}

Participants were asked during and after the workshop to reflect on the capabilities and potential applications of the DBox. The physicality of the instrument was mentioned by several participants. P11: "The way you use the box means you're very close to it. You're touching it, you're inside it, you're very close to try and hear what's going on." P5 commented that the D-Box sound could be influenced by the whole body ("it wasn't just finger based, it wasn't just tapping with my hands it was my whole body, well my whole top half.") P5 also discovered techniques involving loosely connecting wires and hovering the finger just over the sensor. Only P7 asked for a version of the D-Box attached to a computer, citing the ergonomics of hacking the breadboard ("I couldn't control the resistors too well, my fingers were too cumbersome").

Participants viewed the D-Box as complex (P6: "it seemed very simple at first ... but now there are a huge range of com- 


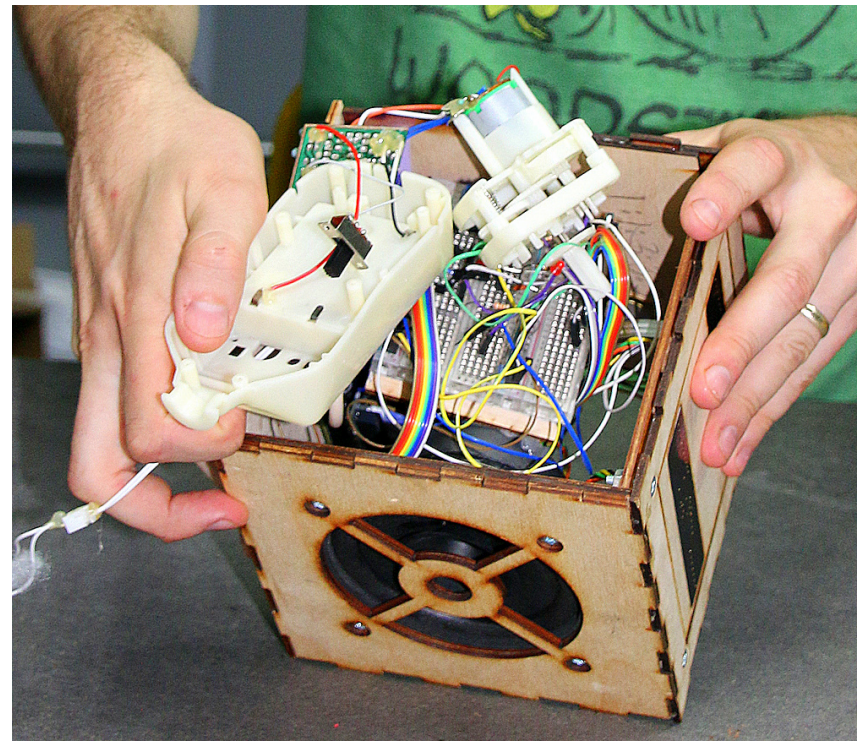

Figure 4. P2's hack, which leaves all wires and other components drifting freely in the box. A spinning motor produces transient connections, leading to chaotic sounds.

binations") and versatile (P8, P12; P1: “It's great. There's literally no cap to what you can do with it."). P8 identified use of the sensors and hacking the interior as two separate ways of playing the instrument. Aside from musical performance, suggested applications included teaching (P14) and music therapy (P12: "It's exciting. It's accessible. It's not scary."). Several participants expressed interest in spending more time with the D-Box beyond the workshop (e.g. P1: "Given a day or two to work out what's going on I could make some cool stuff"; P10: "I would probably like to spend about 5 hours with [workshop leader] and get to know what's going on exactly").

\section{DISCUSSION}

Designing an interface for exploration and play presents unique challenges in that the success of the interface rests entirely on the intrinsic experience of using it. Task completion measures are irrelevant since the task is not well defined. The D-Box, like many playful systems, supports many possible user interpretations. However, in the words of Sengers and Gaver [52], "designing systems to support a rich range of interpretations does not abdicate the designer from responsibility for the eventual success of the system." In this section, we reflect on user reactions to the D-Box and what they suggest about the design process.

\section{Making Meaning}

Traditional HCI holds that a system's affordances should be clearly and unambiguously communicated to the user [44, 52]. We chose to leave the D-Box design ambiguous and open to interpretation, both in its initial presentation to the user and in the function and organisation of the breadboard and its associated circuits. By maintaining ambiguity we can then examine the way that users develop a personal understanding of the instrument [23].

\section{Initial Discovery of Affordances}

Upon receiving a D-Box (with side panels closed), participants quickly identified the two touch sensors as providing the main affordances of the instrument. In the process of understanding their operation, one participant discovered the unusual playing technique of hovering the finger over the sensor, and another developed a muting technique by touching the speaker, but most interaction remained focused on touch position and pressure.

This contrasts with a previous study of a similarly-shaped but highly constrained wooden cube instrument [64]. 10 participants working alone collectively developed a broad range of unusual playing techniques based on hidden affordances [22], including percussion on the case, mechanical filtering of the speaker and application of moisture to the touch sensor. The absence of such discovery here is surprising. It may be that the short time, the fact that participants knew that they would later modify the box, or the social setting where some were copying the interactions of the others nearby, discouraged them from looking beyond the obvious affordances of the two sensors.

\section{Understanding the Parameter Space}

In the process of creating hacks, some participants drew on meaning from other domains, including audio software and colour-sound relationships, to attempt to understand the function of the breadboard. Each interpretation was different, but no user's set of expectations was entirely fulfilled. As previously discussed, this was by deliberate design [40].

In response, we observed a transition from systematic exploration to a mostly trial-and-error approach. The process aligns closely with Morrison's observation [43]: "People ... anticipate things will work in certain ways, and initially may see the work as failing if this doesn't occur. Over time, and by playing with the work, people may arrive at an understanding of the idiosyncratic nature of the work. Once they do this, they 'give over' to enjoying a different type of experience than they had expected." Indeed, participants appeared to enjoy the hacking process. Laughter and spontaneous expressions of amusement were common, and many participants expressed a desire to work with the D-Box for a longer period of time.

\section{Positive and Negative Frustration}

Höök et al. [29] highlight the importance in interactive art contexts of distinguishing between user frustration as an intended outcome and frustration as a result of bad design choices. In the course of developing an understanding of the D-Box, periods of frustration emerged. An instance of intentional frustration was the inability to precisely predict the results of modifying the breadboard, especially when a result lacked sonic variety. There were several signs this was a productive part of the process. It was a transient state associated with certain hacks, which could be changed or reverted. It did not give way to boredom or disengagement; on the contrary, this frustration was often the catalyst for moving to a more exploratory mode of interaction. 
On the other hand, the filter sensor on the D-Box exterior led to frustration indicative of design failure. The similar appearance between it and the pitch sensor (which triggered sounds) set unfulfilled expectations of its operation (a form of false affordance [22]), creating confusion and generally discouraging its use. A possible lesson is that the benefits of ambiguous design do not extend to interfaces which are simply misleading.

\section{Patterns of Exploration: the Cautious Random Walk}

Nearly all participants eventually developed a trial-and-error approach to hacking. We observed a recurring pattern whereby a participant would change one or two things at a time, evaluate the outcome, and then either move forward or revert to the previous state. We call this process a cautious random walk: the generation of ideas is semi-arbitrary, but ideas are then filtered for suitability and interest, with a path to immediate backtracking. When a participant approached a desired sound, exploration often then became more deliberate. From a learning perspective this may be considered behaviourist in nature $[19,61]$.

The cautious random walk relates to Morrison's "speculative play" [42] in its attention to outcomes, but the generation of ideas is more haphazard. It also relates to Tubb and Dixon's model of divergent and convergent navigation of creative parameter spaces [58]: the hacking process is mainly divergent in nature, becoming more convergent (insofar as possible) once a desired point is reached.

This pattern also reflects creative tensions around the topic of control. Alterations to the D-Box were typically unpredictable, but they were repeatable. The instrument is not random, nor does it rely on generative processes which the user can only loosely influence. Participants generally reported not feeling in control (in that they could not predict the results of their actions), but they were aware that their actions were the catalyst for change, and participants retained editorial authority on whether or not to accept an outcome.

This creative tension, we suggest, was an important motivator in the D-Box workshops. Where boredom results from interactive systems that are overly simple [43], too slow or apparently random [29], participants described the D-Box experience as exciting. P7: "But [not being in control] is part of the fun of it isn't it, it's a bit like chance music I guess. You can't predict what will happen and I think that's quite exciting." P17: "I think that kind of makes it exciting, not knowing what to expect."

Pike et al. [48] draw a contrast between the "extent of voluntary control" and "extent of self-awareness" of how conscious the user is of the control process. The D-Box may provide a combination of high (nearly complete) voluntary control with medium-to-low self-awareness.

It is also worth noting that the workshops are effectively a community of practice [62] and knowledge in this setting is co-constructed. We recognise that conversations and interactions played a role here [8] and that knowledge was constructed from both an individual and group perspective [57].

\section{Comparison to Software Tools}

The cautious random walk process appears to emerge from the hardware-hacking nature of the D-Box, where there is no opportunity to save and restore settings, nor any undo feature aside from remembering what came before. In addition to the tension of control, the lack of automatic undo creates a tension between staying with a favoured system state or taking a risk to see what might be around the next corner.

Studies of software interfaces show a distinctly different pattern. Tubb and Dixon [58] observe that in a musical interface consisting of multiple on-screen sliders, users initially performed large adjustments (exploring the range of the space) which became progressively smaller over time (exploring the details). Ekeus et al. [17] show that in a smartphone-based 2D music parameter navigation task, users tend to gravitate to points on the extreme ends of the range. In software interfaces, there is typically no cost to moving a control across its entire range, so it is a natural reaction to explore the limits first and then settle on a desired setting.

The D-Box stymies any attempt to discover the limits of its parameter space, because the space is nonlinear and potentially discontinuous, and because the parameters are interdependent. Its physical nature also means that time and effort scale with the complexity of the change, and the lack of undo raises the cost of a false move.

\section{Ownership and Personalisation}

Some participants developed a personal attachment to their original D-Box. This was observed in the strong negative reactions by 5 participants to receiving a new D-Box, and to comments by some that they missed their original one. Another frequent comment was that the new D-Box was harder to understand than their own.

This finding is interesting because every D-Box began with the same configuration, and the hacks were completed over a relatively short period of time. Furthermore, participants often acknowledged that they did not understand the process by which they had created their original hack. It cannot be the case, then, that participants had aimed to create a particular hack from the start of the workshop. Instead, our observation may suggest that the creative filtering process (choosing which hacks to accept and which to revert or discard) was responsible for fostering personal attachment.

On the other hand, creative filtering and remixing processes have been studied in online maker communities [45] without the emergence of a similar sense of personal attachment. Perhaps control intimacy rather than the modification process is responsible for this effect (see P11's comment in Reflections on the D-Box). The role of physical effort and craft knowledge in leading to attachment has been noted in other artistic domains [15]. This view is also supported by our earlier study of a highly constrained musical instrument of similar physical design [64], where we observed a similar sense of attachment even though the instruments were nominally identical and not modifiable. Perhaps like a favourite guitar, a performer becomes attuned to the smallest idiosyncrasies of the 
instrument, making any change uncomfortable. Further study is needed to better understand this effect.

\section{Long-Term Use}

The workshops in this paper were intended to capture the emergent processes of exploratory play in the first few hours of encountering an unfamiliar instrument. Longitudinal use of the D-Box for public musical performance has been previously studied $[63,65]$. In these cases, several performers made more extensive, systematic modifications, including adding buttons or potentiometers to the case which affected the circuits on the matrix. In other cases, performers practiced specific modifications to the wiring which they performed at a particular point in their piece.

Our studies have focused on either short-term exploratory play or the outputs of extended practice, but it is likely that the D-Box allows a smooth transition from one mode to the other. P9 commented: "I feel that it is a hackable thing initially, but then you want to showcase what it is you have.... If you gave me 5 hours then it would be cool to show it around." Evidence that the D-Box produces sustained interest also comes from other workshops (not in this study) where at the end, some participants have opted to buy the instrument they used.

\section{CONCLUSION}

We observed several themes and patterns in participants' explorations of the D-Box, some of which confirm prior literature, especially around the value of ambiguity in design, and others which stand in contrast to other studies.

The D-Box and its use in these workshops links at least partially to all three of Gaver's opening assumptions for ludic design [24]: "Promote curiosity, exploration and reflection"; "De-emphasise the pursuit of external goals"; and "Maintain openness and ambiguity." The design of the D-Box is left open to interpretation, and its possible range of sonic outputs is impossible to discover except through extended exploration. Participants showed consistent curiosity and engagement, and we observed a recurring pattern of exploration we call the cautious random walk, which alternated between open exploration (choosing the next hack) and reflection (evaluating its quality in relation to previous hacks).

The design of the D-Box is neither user-centred nor participatory, and might even be considered adversarial: we sought to create a device whose operation defied easy categorisation, while still placing all control in the hands of the user rather than in internal generative processes. This design process aligns with Sengers and Gaver's critique of user-centred design as seeking to fix a single interpretation instead of allowing ambiguity [52].

The patterns of exploration in the D-Box appear different than those in creative software interfaces, suggesting that imposing a cost on changes (through effort and lack of an undo) changes how participants explore the device. We also saw some participants develop a sense of personal attachment to their D-Boxes which appears to differ from reactions to either software or interactive art installations. In the preceding section we speculate on the reasons for this effect.

\section{Tensions in Exploratory Play}

Our findings point to several sources of creative tension in exploratory play:

1. Control: there may be a tipping point of how much lack of control users are willing to accept, as we observed from the differing reactions before and after the D-Box swap. There may also be a tension between knowing that one is in control and knowing how that control works, with the first of these being sufficient to maintain engagement in an activity.

2. Unpredictability: a predictable device lends itself to systematic exploration, where an unpredictable device encourages trial-and-error exploration. But where apparently random behaviour may lead to boredom or disengagement, unpredictable but consistent behaviour can be a source of curiosity.

3. Frustration: following [29], user frustration can provide incentive or disincentive to continue interacting with a device, depending on the context. Design choices can be responsible for one or both forms of frustration.

4. Ownership: users of a modifiable device may begin to feel a sense of personal attachment: here, a transition from "a D-Box" to "my D-Box." This might be expected in a goaldirected participatory design process, but it also emerges through exploratory modifications.

\section{Designing for Exploratory Play: Recommendations}

We distil our findings into a set of design recommendations for supporting exploratory play which expand on [24]:

1. Defy conventional affordances or make the affordances ambiguous, but don't make them deliberately misleading.

2. Grant permission to explore by making the device appear safely modifiable without damage.

3. Give full control but only partial predictability. Make the user aware that their actions determine the output, but don't divulge the entire space of possibilities at the first encounter.

We add a further suggestion for designing activities and processes by which users encounter unfamiliar devices:

4. Perturb the process. Add a step to the activity which disrupts any points of stasis that have emerged. Here, swapping D-Boxes gave participants license to take a less cautious approach to hacking.

As a final note on future work, we intend to explore the recent notion of accountable artefacts [3], where everyday things can be flexibly mapped to their digital record so as to tell different stories of provenance, history and ongoing use - a kind of social self-documentation. Linking individual D-Boxes to digital records may enable us to address the particular tension between saving and exploring new hacks as well as providing a mechanism for users to share hacks. 


\section{REFERENCES}

1. Juha Arrasvuori, Hannu Korhonen, and Kaisa Väänänen-Vainio-Mattila. 2010. Exploring playfulness in user experience of personal mobile products. In Proceedings of the 22nd Conference of the Computer-Human Interaction Special Interest Group of Australia on Computer-Human Interaction. ACM, 88-95.

2. Janet B Arthurs. 2007. A juggling act in the classroom: Managing different learning styles. Teaching and learning in nursing 2, 1 (2007), 2-7.

3. Steve Benford, Adrian Hazzard, Alan Chamberlain, Kevin Glover, Chris Greenhalgh, Liming Xu, Michaela Hoare, and Dimitrios Darzentas. 2016. Accountable Artefacts: the Case of the Carolan Guitar. In Proceedings of the SIGCHI Conference on Human Factors in Computing Systems.

4. Ben Bengler and Nick Bryan-Kinns. 2013. Designing collaborative musical experiences for broad audiences. In Proceedings of the 9th ACM Conference on Creativity \& Cognition.

5. Tina Blaine and Sidney Fels. 2003. Contexts of Collaborative Musical Experiences. In Proceedings of the International Conference on New Interfaces for Musical Expression.

6. Benjamin S Bloom. 1987. A Response to Slavin's Mastery Learning Reconsidered. Review of Educational Research 57, 4 (1987), 507-8.

7. John Bowers and Phil Archer. 2005. Not hyper, not meta, not cyber but infra-instruments. In Proceedings of the International Conference on New Interfaces for Musical Expression.

8. Eva Buchinger and Bernard Scott. 2010. Comparing conceptions of learning: Pask and Luhmann. Constructivist Foundations 5, 3 (2010), 109-120.

9. Nicolas Collins. 2008. A Solder's Tale: Putting the 'Lead' Back in 'Lead Users'. Pervasive Computing 7 (2008), 32-38. Issue 3.

10. Nicolas Collins. 2009. Handmade Electronic Music: The Art of Hardware Hacking (second ed.). Routledge, New York.

11. Andy Crabtree, Tom Rodden, Terry Hemmings, and Steve Benford. 2003. Finding a Place for UbiComp in the Home. In UbiComp 2003: Ubiquitous Computing. Springer, 208-226.

12. Palle Dahlstedt, Per Anders Nilsson, and Gino Robair. 2015. The Bucket System - a computer mediated signaling system for group improvisation. In Proceedings of the International Conference on New Interfaces for Musical Expression.

13. Alan Dix. 2007. Designing for appropriation. In Proc. British HCI Group Conf. on People and Computers.
14. Christopher Dobrian and Daniel Koppelman. 2006. The ' $E$ ' in NIME: musical expression with new computer interfaces. In Proceedings of the International Conference on New Interfaces for Musical Expression.

15. Peter Dormer. 1994. The art of the maker. Thames and Hudson London.

16. Paul Dourish. 2001. Where the action is: the foundations of embodied interaction. MIT press.

17. Henrik Ekeus, Samer A Abdallah, Mark D Plumbley, and Peter W McOwan. 2012. The Melody Triangle: Exploring Pattern and Predictability in Music. In Proc. Musical Metacreation (MUME), 1st International Workshop on. 35-42.

18. Yrjo Engeström. 2000. Activity theory and the social construction of knowledge: A story of four umpires. Organization 7, 2 (2000), 301-310.

19. Qais Faryadi. 2007. Behaviorism and the Construction of Knowledge. Online Submission (2007).

20. Jenny Fleming and Lesley Ferkins. 2010. The use of action learning strategies for cooperative education or work-integrated learning projects. Learning and Teaching in Higher Education Journal 4, 1 (2010), 1-4.

21. William Gaver. 2002. Designing for Homo Ludens. I3 Magazine 12 (2002).

22. William W Gaver. 1991. Technology affordances. In Proceedings of the SIGCHI conference on Human factors in computing systems. ACM, 79-84.

23. William W. Gaver, Jacob Beaver, and Steve Benford. 2003. Ambiguity As a Resource for Design. In Proceedings of the SIGCHI Conference on Human Factors in Computing Systems (CHI '03). ACM, New York, NY, USA, 233-240. DOI : http://dx.doi.org/10.1145/642611.642653

24. William W. Gaver, John Bowers, Andrew Boucher, Hans Gellerson, Sarah Pennington, Albrecht Schmidt, Anthony Steed, Nicholas Villars, and Brendan Walker. 2004. The Drift Table: Designing for Ludic Engagement. In CHI '04 Extended Abstracts on Human Factors in Computing Systems (CHI EA '04). ACM, New York, NY, USA, 885-900. DOI : http://dx.doi.org/10.1145/985921.985947

25. Steven Gelineck and Stefania Serafin. 2012. Longitudinal evaluation of the integration of digital musical instruments into existing compositional work processes. Journal of New Music Research 41, 3 (2012), 259-276.

26. Reed Ghazala. 2005. Circuit Bending: Build Your Own Alien Instruments. Wiley.

27. Michael Gurevich, Paul Stapleton, and Adnan Marquez-Borbon. 2010. Style and Constraint in Electronic Musical Instruments. In Proceedings of the International Conference on New Interfaces for Musical Expression. 
28. Kristina Höök. 2006. Designing familiar open surfaces. In Proceedings of the 4th Nordic conference on Human-computer interaction: changing roles. ACM, 242-251.

29. Kristina Höök, Phoebe Sengers, and Gerd Andersson. 2003. Sense and Sensibility: Evaluation and Interactive Art. In Proceedings of the SIGCHI Conference on Human Factors in Computing Systems (CHI '03). ACM, New York, NY, USA, 241-248. DOI : http://dx.doi.org/10.1145/642611.642654

30. Hilary Hutchinson, Wendy Mackay, Bo Westerlund, Benjamin B. Bederson, Allison Druin, Catherine Plaisant, Michel Beaudouin-Lafon, Stéphane Conversy, Helen Evans, Heiko Hansen, Nicolas Roussel, and Björn Eiderbäck. 2003. Technology Probes: Inspiring Design for and with Families. In Proceedings of the SIGCHI Conference on Human Factors in Computing Systems (CHI '03). ACM, New York, NY, USA, 17-24. DOI : http://dx.doi.org/10.1145/642611.642616

31. Sergi Jordà. 2004. Instruments and Players: Some Thoughts on Digital Lutherie. J. New Music Research 33 (2004), 321-341. Issue 3.

32. Sergi Jordà and Sebastián Mealla. 2014. A methodological framework for teaching, evaluating and informing NIME design with a focus on expressiveness and mapping. In Proceedings of the International Conference on New Interfaces for Musical Expression, Vol. 30. 233-238.

33. Chris Kiefer, Nick Collins, and Geraldine Fitzpatrick. 2008. HCI methodology for evaluating musical controllers: A case study. In Proceedings of NIME, Vol. 8.

34. J-L Le Moigne. 2011. From Jean Piaget to Ernst von Glasersfeld: An epistemological itinerary in review. Constructivist Foundations 6, 2 (2011), 152-156.

35. Silvia Lindtner, Garnet D. Hertz, and Paul Dourish. 2014. Emerging Sites of HCI Innovation: Hackerspaces, Hardware Startups \&\#38; Incubators. In Proceedings of the SIGCHI Conference on Human Factors in Computing Systems (CHI '14). ACM, New York, NY, USA, 439-448. DOI : http://dx.doi.org/10.1145/2556288.2557132

36. Thor Magnusson. 2010. Designing Constraints: Composing and Performing with Digital Musical Systems. Computer Music J. 34 (2010), 62-73. Issue 4.

37. Adnan Marquez-Borbon, Michael Gurevich, A Cavan Fyans, and Paul Stapleton. 2011. Designing digital musical interactions in experimental contexts. Proceedings of the International Conference on New Interfaces for Musical Expression (2011).

38. A. McPherson and Y. Kim. 2012. The problem of the second performer: building a community around an augmented instrument. Computer Music Journal 34, 4 (2012).
39. Andrew McPherson and Victor Zappi. 2015a. An environment for submillisecond-latency audio and sensor processing on BeagleBone Black. In Proc. AES 138th Conv.

40. Andrew McPherson and Victor Zappi. 2015b. Exposing the Scaffolding of Digital Instruments with Hardware-Software Feedback Loops. In Proceedings of the International Conference on New Interfaces for Musical Expression.

41. Fabio Morreale, Antonella De Angeli, Raul Masu, Paolo Rota, and Nicola Conci. 2014. Collaborative creativity: The Music Room. Personal and Ubiquitous Computing 18, 5 (2014), 1187-1199.

42. Ann Morrison, Stephen Viller, and Peta Mitchell. 2011. Building Sensitising Terms to Understand Free-play in Open-ended Interactive Art Environments. In Proceedings of the SIGCHI Conference on Human Factors in Computing Systems (CHI'11). ACM, New York, NY, USA, 2335-2344. DOI : http://dx.doi.org/10.1145/1978942.1979285

43. Ann J. Morrison, Peta Mitchell, and Margot Brereton. 2007. The Lens of Ludic Engagement: Evaluating Participation in Interactive Art Installations. In Proceedings of the 15th International Conference on Multimedia (MULTIMEDIA '07). ACM, New York, NY, USA, 509-512. DOI : http://dx.doi.org/10.1145/1291233.1291358

44. Donald A Norman. 1988. The psychology of everyday things. Basic books.

45. Lora Oehlberg, Wesley Willett, and Wendy E. Mackay. 2015. Patterns of Physical Design Remixing in Online Maker Communities. In Proceedings of the 33rd Annual ACM Conference on Human Factors in Computing Systems (CHI'15). ACM, New York, NY, USA, 639-648. DOI : http://dx.doi.org/10.1145/2702123.2702175

46. Sile O'modhrain. 2011. A framework for the evaluation of digital musical instruments. Computer Music Journal 35, 1 (2011), 28-42.

47. Jon Ord. 2012. John Dewey and Experiential Learning: Developing the theory of youth work. Youth \& Policy 108 (2012), 55-72.

48. Matthew Pike, Richard Ramchurn, Steve Benford, and Max L. Wilson. 2016. \#Scanners: Exploring the Control of Adaptive Films using Brain-Computer Interaction. In Proceedings of the SIGCHI Conference on Human Factors in Computing Systems.

49. Cornelius Poepel. 2005. On interface expressivity: a player-based study. In Proceedings of the 2005 conference on New interfaces for musical expression. National University of Singapore, 228-231.

50. David Roedl, Shaowen Bardzell, and Jeffrey Bardzell. 2015. Sustainable Making? Balancing Optimism and Criticism in HCI Discourse. ACM Trans. Comput.-Hum. 
Interact. 22, 3, Article 15 (June 2015), 27 pages. DOI : http://dx.doi.org/10.1145/2699742

51. J. Rogers. 2001. Honey and Mumford's Learning Styles. In Adults Learning.

52. Phoebe Sengers and Bill Gaver. 2006. Staying open to interpretation: engaging multiple meanings in design and evaluation. In Proceedings of the 6th Conference on Designing Interactive Systems. ACM, 99-108.

53. Jin-min Seok, Jong-bum Woo, and Youn-kyung Lim. 2014. Non-finito Products: A New Design Space of User Creativity for Personal User Experience. In Proceedings of the SIGCHI Conference on Human Factors in Computing Systems (CHI'14). ACM, New York, NY, USA, 693-702. DOI :

http://dx.doi.org/10.1145/2556288.2557222

54. Stephen Swailes and Barbara Senior. 1999. The dimensionality of Honey and Mumford's learning styles questionnaire. International Journal of Selection and Assessment 7, 1 (1999), 1-11.

55. Atau Tanaka, Baptiste Caramiaux, and Norbert Schnell. 2013. MubuFunkScatShare: gestural energy and shared interactive music. In Proc. CHI Ext. Abstracts.

56. Joshua G. Tanenbaum, Amanda M. Williams, Audrey Desjardins, and Karen Tanenbaum. 2013. Democratizing Technology: Pleasure, Utility and Expressiveness in DIY and Maker Practice. In Proceedings of the SIGCHI Conference on Human Factors in Computing Systems (CHI '13). ACM, New York, NY, USA, 2603-2612. DOI : http://dx.doi.org/10.1145/2470654.2481360

57. Peter C Taylor. 2004. Radical constructivism in action: Building on the pioneering work of Ernst von Glasersfeld. Science Education 88, 1 (2004), 149-152.

58. Robert Tubb and Simon Dixon. 2014. The Divergent Interface: Supporting Creative Exploration of Parameter Spaces. In Proceedings of the International Conference on New Interfaces for Musical Expression, Vol. 1257.

59. M. M. Wanderley and N. Orio. 2002. Evaluation of Input Devices for Musical Expression: Borrowing Tools from HCI. Computer Music Journal 26 (2002), 62-76. Issue 3.

60. Darren Webb. 2010. Paulo Freire and 'the need for a kind of education in hope. Cambridge Journal of Education 40, 4 (2010), 327-339.

61. Mary Anne Weegar and Dina Pacis. 2012. A Comparison of Two Theories of Learning-Behaviorism and Constructivism as applied to Face-to-Face and Online Learning. In Proceedings E-Leader Conference, Manila.

62. Etienne Wenger. 1999. Communities of practice: Learning, meaning, and identity. Cambridge University Press.
63. Victor Zappi and Andrew McPherson. 2014a. Design and Use of a Hackable Digital Instrument. In Proceedings of the International Conference on Live Interfaces.

64. Victor Zappi and Andrew McPherson. 2014b. Dimensionality and appropriation in digital musical instrument design. In Proceedings of the International Conference on New Interfaces for Musical Expression.

65. Victor Zappi and Andrew McPherson. 2015. The D-Box: How to Rethink a Digital Musical Instrument. In Proceedings of the International Symposium on Electronic Art. 